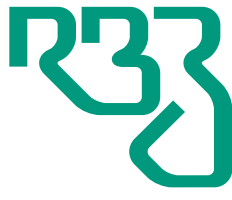

Revista Brasileira de Zootecnia

Brazilian Journal of Animal Science ISSN 1806-9290 www.rbz.org.br

\section{Increasing feed allowance in low-fish meal diets allows for a reduction in dietary methionine for juvenile Litopenaeus vannamei raised in green-water tanks}

\author{
Felipe Nobre Façanha ${ }^{1}$ iD, Hassan Sabry-Neto ${ }^{1}$, Adhemar Rodrigues de \\ Oliveira-Neto $^{2}$ (iD, Claudia Figueiredo-Silva ${ }^{3}$, Alberto Jorge Pinto Nunes ${ }^{1^{*}}$ \\ ${ }^{1}$ Universidade Federal do Ceará, Instituto de Ciências do Mar, Fortaleza, CE, Brasil. \\ ${ }^{2}$ Evonik Brasil Ltda, São Paulo, SP, Brasil. \\ ${ }^{3}$ Evonik Nutrition \& Care GmbH, Hanau, Germany.
}

*Corresponding author:

alberto.nunes@ufc.br

Received: August 21, 2018

Accepted: July 2, 2019

How to cite: Façanha, F. N.; Sabry-Neto, H.; Oliveira-Neto, A. R.; Figueiredo-Silva, C. and Nunes, A. J. P. 2019. Increasing feed allowance in low-fish meal diets allows for a reduction in dietary methionine for juvenile Litopenaeus vannamei raised in green-water tanks. Revista Brasileira de Zootecnia 48:e20180198. https://doi.org/10.1590/rbz4820180198

Copyright: This is an open access article distributed under the terms of the Creative Commons Attribution License (http://creativecommons.org/licenses/by/4.0/) which permits unrestricted use, distribution, and reproduction in any medium, provided the original work is properly cited.

\begin{abstract}
A 10-week study was conducted to evaluate the effect of feed allowance and graded levels of dietary methionine (Met) on growth performance of Litopenaeus vannamei. Juvenile shrimp of $1.83 \pm 0.14 \mathrm{~g}$ were stocked in 42 outdoor green-water tanks of $1 \mathrm{~m}^{3}$ under 120 shrimp $\mathrm{m}^{-2}$. Animals were fed under two feed allowances, regular and $30 \%$ in excess. Five diets with $30 \mathrm{~g} \mathrm{~kg}^{-1}$ fishmeal were designed to contain $318 \pm 2 \mathrm{~g} \mathrm{~kg}^{-1}$ crude protein and a minimum amount of protein-bound Met. To achieve graded levels of dietary Met, a control diet with $4.6 \mathrm{~g} \mathrm{~kg}^{-1}$ Met or $8.9 \mathrm{~g} \mathrm{~kg}^{-1}$ methionine + cysteine $(\mathrm{M}+\mathrm{C})$ was supplemented with $1.2,2.2,3.2$, and $4.2 \mathrm{~g} \mathrm{~kg}^{-1}$ of DL-methionylDL-methionine to result in total dietary Met of 5.6, 6.9, 7.9, and $9.2 \mathrm{~g} \mathrm{~kg}^{-1}(10.0,11.2$, 12.1 , and $13.5 \mathrm{~g} \mathrm{~kg}^{-1} \mathrm{M}+\mathrm{C}$, respectively). A final survival of $86.5 \pm 3.6 \%$ was reached with no significant influence from feed allowance or dietary Met. Feed inputs significantly affected apparent feed intake, weekly shrimp growth, final body weight (BW), and gained yield. Larger meals and a higher dietary Met had no impact on feed conversion ratio. There was a significant interaction between feed allowance and Met over shrimp BW. By feeding animals in excess, BW was enhanced at $6.9 \mathrm{~g} \mathrm{~kg}^{-1}$ Met. A dietary Met of $7.9 \mathrm{~g} \mathrm{~kg}^{-1}$ was required to achieve a maximum BW under a regular feed allowance. Thus, shrimp required less amounts of dietary Met to maximize BW when higher feed inputs were delivered. Our findings demonstrate a sparing effect of dietary Met for L. vannamei when a higher feed allowance is adopted. Shrimp farmers should consider adjusting feed allowance to dietary Met to maximize shrimp growth performance and yield.
\end{abstract}

Keywords: amino acid supplementation, ration size, shrimp, supplementation

\title{
Introduction
}

In marine shrimp culture, feed inputs are calculated to maximize shrimp growth and feed efficiency while increasing crop turn-over. Daily rations are adjusted using feeding tables in combination with estimates of shrimp growth, survival, feed conversion ratio (FCR), and feed intake. In rearing systems where naturally occurring food sources are more abundant, feed inputs may be reduced to enhance FCR and feed costs (Nunes et al., 1997; Cho et al., 2001; Cho and Lovell, 2002; Venero et al., 2007).

There also appears to exist an association between feed inputs and dietary nutrient content. In juvenile whiteleg shrimp, Litopenaeus vannamei, Nunes et al. (2006) restrained feed allowance by $25 \%$ below apparent satiation with no negative effect on shrimp performance. Venero et al. (2007) also demonstrated that an increase in the dietary protein content from 322 to $425 \mathrm{~g} \mathrm{~kg}^{-1}$ allows for a $25 \%$ reduction in feed inputs without affecting the growth performance of L. vannamei. 
Recent studies indicate that growth stage, stocking density, salinity, and water exchange regime have an effect on the amino acid requirements of penaeid shrimp (Duy et al., 2012; Façanha et al., 2016, 2018; Liu et al., 2014). However, compared with other production animals (e.g., swine and poultry), studies evaluating the interactions of non-dietary factors and nutritional requirements of farmed shrimp are scarce. In shrimp farming, experimental green-water tank culture systems better resemble the dynamics of commercial culture ponds. Green-water systems operate outdoors for exposure to sunlight, which drives photosynthesis, development of naturally occurring food items, and daily variations in water quality. We have conducted several studies under these conditions (Façanha et al., 2016, 2018; Nunes et al., 2019a,b), which showed that the dietary methionine (Met) content strongly interacts with shrimp stocking density, natural food availability, water exchange, and minimum level of dietary crude protein content for optimal shrimp growth. The higher the stocking density, the less available natural food becomes and the higher is the amount of dietary Met required to maximize shrimp growth (Façanha et al., 2016). Conversely, limiting water exchange in a green-water system spares the requirement of dietary Met for juvenile whiteleg shrimp (Façanha et al., 2018).

The objective of the present study was to evaluate the effect of feed allowance and graded levels of dietary methionine on growth performance of juvenile Litopenaeus vannamei raised under a green-water rearing system.

\section{Material and Methods}

The study was conducted in experimental outdoor tank facilities located in the city of Eusébio, CE, Brazil $\left(3^{\circ} 50^{\prime} 01.55^{\prime \prime} \mathrm{S}\right.$ and $\left.38^{\circ} 25^{\prime} 22.74^{\prime \prime} \mathrm{W}\right)$. All procedures were performed in compliance with relevant laws and institutional guidelines, including those related to animal welfare.

The study consisted in the evaluation of five diets formulated to contain graded levels of dietary methionine (Met), from 4.6 to $9.2 \mathrm{~g} \mathrm{~kg}^{-1}$ ( $\mathrm{g} \mathrm{kg}^{-1}$ of the diet, on a dry matter basis, DM). Juvenile shrimp were stocked in 42 outdoor tanks of $1 \mathrm{~m}^{3}$ in a completely randomized design under 120 animals m $\mathrm{m}^{-2}$. Shrimp were fed under two feed allowances, regular and 30\% in excess. Four rearing tanks were randomly assigned for each set of diet and feed allowance, except shrimp fed diets containing $4.6 \mathrm{~g} \mathrm{~kg}^{-1}$ total Met content, which operated with five tanks. Animals were raised for 70 days.

Five experimental diets were designed with a minimum inclusion of fishmeal and other marine ingredients (Table 1). The dietary inclusion of salmon byproduct meal, squid meal, and salmon oil were locked at 30.0,10.0, and $31.2 \mathrm{~g} \mathrm{~kg}^{-1}$ of the diet (as is basis), respectively. Squid meal was added to all diets to confer feed attractability. Soybean meal was the major protein component in the formulas included at $338.0 \pm 2.4 \mathrm{~g} \mathrm{~kg}^{-1}$ (mean \pm standard deviation). The amount of wheat flour slightly changed among experimental diets (from 438.3 to $439.2 \mathrm{~g} \mathrm{~kg}^{-1}$ ) to account for the different inclusion of supplemental amino acids. Soy lecithin, cholesterol, and fish oil were included to meet shrimp nutrient requirements for phospholipids, cholesterol, and n-3 LC-PUFA (omega-3 long-chain polyunsaturated fatty acids), respectively. All feeds were formulated to contain a minimum of $390 \mathrm{mg} \mathrm{kg}^{-1}$ of ascorbic acid.

Diets contained a mean crude protein (CP) content of $318 \pm 1.8 \mathrm{~g} \mathrm{~kg}^{-1}$ (Table 2). To achieve graded levels of dietary Met, a basal diet was first designed to contain a minimum level of Met originating only from intact sources. From this diet, four nearly similar diets were supplemented with a dipeptide, DL-methionyl-DL-methionine (AQUAVI ${ }^{\circledR}$ Met-Met, Evonik Nutrition \& Care GmbH, Hanau, Germany) at $0.1,1.2,2.2,3.2$, and $4.2 \mathrm{~g} \mathrm{~kg}^{-1}$ of the diet (as is) to achieve a total dietary Met of 4.6, 5.6, 6.9, 7.9, and $9.2 \mathrm{~g} \mathrm{~kg}^{-1}$ (DM basis), with a corresponding methionine + cysteine (Met+Cys) level of 8.9, 10.0, 11.2, 12.1 , and $13.5 \mathrm{~g} \mathrm{~kg}^{-1}$, respectively.

To maximize protein utilization, all diets were formulated on an ideal protein concept using lysine as the first limiting and reference amino acid (NRC, 2011). Therefore, diets were also supplemented with L-Lysine, L-Threonine, and L-Arginine. The following Lys:EAA (essential amino acid) ratios were adopted (NRC, 2011): 100 Lys:67 Thr (threonine), and 100 Lys:95 Arg (arginine). Dietary 
variation (coefficient of variation, CV) of all amino acids, except Met and Met+Cys, were maintained at less than $3 \%$. Diets were lab-extruded and manufactured following procedures described in Nunes et al. (2011).

Experimental diets were analyzed for dry matter (drying in a convection oven for $24 \mathrm{~h}$ at $105^{\circ} \mathrm{C}$ ) and CP (Kjeldahl method of nitrogen estimation) following standard methods (AOAC, 2002). Determination of dietary amino acid concentration followed procedures described by Figueiredo-Silva et al. (2015).

Post-larvae (PL) of L. vannamei were obtained from a commercial hatchery (Aquatec Aquacultura Ltda., Canguaretama, Brazil) and nursery-reared in the laboratory from PL10 to juvenile size. A total of 5,376 shrimp of $1.83 \pm 0.14 \mathrm{~g}(\mathrm{CV}=7.7 \%)$ were individually weighed for culling and stocked in the experimental rearing system.

The rearing system and water preparation were the same as adopted by Nunes et al. (2011), Façanha et al. $(2016,2018)$, and Nunes et al. $(2019 a, b)$. Outdoor tanks were equipped with their own water inlet and outlet, aeration system, and feeding tray. All tanks were round, blue in color,

Table 1 - Ingredient composition of diets

\begin{tabular}{|c|c|c|c|c|c|}
\hline \multirow{2}{*}{ Ingredient $\left(\mathrm{g} \mathrm{kg}^{-1}\right)$} & \multicolumn{5}{|c|}{ Diet composition ( $\mathrm{g} \mathrm{kg}^{-1}$, as is) } \\
\hline & 4.6 & 5.6 & 6.9 & 7.9 & 9.2 \\
\hline Soybean meal $^{1}$ & 341.0 & 339.5 & 338.0 & 336.5 & 335.0 \\
\hline Wheat flour ${ }^{2}$ & 438.3 & 438.4 & 438.6 & 438.9 & 439.2 \\
\hline Salmon meal ${ }^{3}$ & 30.0 & 30.0 & 30.0 & 30.0 & 30.0 \\
\hline Wheat bran & 30.0 & 30.0 & 30.0 & 30.0 & 30.0 \\
\hline Wheat gluten ${ }^{4}$ & 20.0 & 20.0 & 20.0 & 20.0 & 20.0 \\
\hline Squid meal ${ }^{5}$ & 10.0 & 10.0 & 10.0 & 10.0 & 10.0 \\
\hline Salmon oil & 31.2 & 31.2 & 31.2 & 31.2 & 31.2 \\
\hline Soybean oil & 0.8 & 0.9 & 0.9 & 0.9 & 1.0 \\
\hline Soy lecithin & 36.9 & 36.9 & 36.9 & 36.9 & 36.9 \\
\hline Dicalcium phosphate $^{6}$ & 20.0 & 20.0 & 20.0 & 20.0 & 20.0 \\
\hline Vitamin-mineral premix ${ }^{7}$ & 10.0 & 10.0 & 10.0 & 10.0 & 10.0 \\
\hline Magnesium sulfate & 8.5 & 8.5 & 8.6 & 8.6 & 8.6 \\
\hline L-Lysine ${ }^{8}$ & 11.4 & 11.5 & 11.6 & 11.7 & 11.8 \\
\hline DL-Met-Met ${ }^{9}$ & 0.1 & 1.2 & 2.2 & 3.2 & 4.2 \\
\hline L-Threonine $^{10}$ & 3.6 & 3.7 & 3.7 & 3.7 & 3.7 \\
\hline L-Arginine $^{11}$ & 2.1 & 2.1 & 2.2 & 2.3 & 2.3 \\
\hline Synthetic binder ${ }^{12}$ & 5.0 & 5.0 & 5.0 & 5.0 & 5.0 \\
\hline Cholesterol $^{13}$ & 0.7 & 0.7 & 0.7 & 0.7 & 0.7 \\
\hline Vitamin $C^{14}$ & 0.4 & 0.4 & 0.4 & 0.4 & 0.4 \\
\hline
\end{tabular}

${ }^{1}$ Bunge Alimentos S.A. (Luiz Eduardo Magalhães, Brazil): $525.8 \mathrm{~g} \mathrm{~kg}^{-1}$ crude protein (CP, on a total dry matter basis), $31.0 \mathrm{~g} \mathrm{~kg}^{-1} \mathrm{lysine} \mathrm{Lys}$ ), $6.9 \mathrm{~g} \mathrm{~kg}^{-1}$ methionine (Met), $14.6 \mathrm{~g} \mathrm{~kg}^{-1}$ methionine+cysteine (Met+Cys), $20.5 \mathrm{~g} \mathrm{~kg}^{-1}$ threonine (Thr), $37.6 \mathrm{~g} \mathrm{~kg}^{-1} \mathrm{arginine}^{-(A r g)}$.

$133.7 \mathrm{~g} \mathrm{~kg}^{-1} \mathrm{CP}, 2.8 \mathrm{~g} \mathrm{~kg}^{-1} \mathrm{Lys}, 2.1 \mathrm{~g} \mathrm{~kg}^{-1} \mathrm{Met}, 5.2 \mathrm{~g} \mathrm{~kg}^{-1} \mathrm{Met}+\mathrm{Cys}, 3.5 \mathrm{~g} \mathrm{~kg}^{-1} \mathrm{Thr}, 5.3 \mathrm{~g} \mathrm{~kg}^{-1} \mathrm{Arg}$.

${ }^{3}$ Pesquera Pacific Star S.A. (Puerto Montt, Chile): $701.4 \mathrm{~g} \mathrm{~kg}^{-1} \mathrm{CP}, 45.8 \mathrm{~g} \mathrm{~kg}^{-1} \mathrm{Lys}, 17.3 \mathrm{~g} \mathrm{~kg}^{-1} \mathrm{Met}, 22.9 \mathrm{~g} \mathrm{~kg}^{-1} \mathrm{Met} \mathrm{Cys}, 27.1 \mathrm{~g} \mathrm{~kg}^{-1} \mathrm{Thr}, 41.8 \mathrm{~g} \mathrm{~kg}^{-1} \mathrm{Arg}$

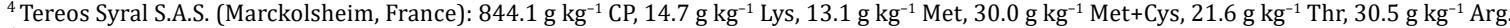

$5921.1 \mathrm{~g} \mathrm{~kg}^{-1} \mathrm{CP}, 56.1 \mathrm{~g} \mathrm{~kg}^{-1} \mathrm{Lys}, 25.9 \mathrm{~g} \mathrm{~kg}^{-1} \mathrm{Met}, 35.1 \mathrm{~g} \mathrm{~kg}^{-1} \mathrm{Met}+\mathrm{Cys}, 36.9 \mathrm{~g} \mathrm{~kg}^{-1} \mathrm{Thr}, 63.0 \mathrm{~g} \mathrm{~kg}^{-1} \mathrm{Arg}^{-}$

${ }^{6}$ Serrana Foscálcio20 (Bunge Fertilizantes S/A, Cubatão, Brazil): $20.5 \%$ calcium, $20.2 \%$ total phosphorous.

${ }^{7}$ Rovimix $^{\circledast}$ Camarões Intensivo (DSM Produtos Nutricionais Brasil Ltda., São Paulo, Brazil); guaranteed levels per kg of product: vitamin A, 1,250,000 IU; vitamin D3, 350,000 IU; vitamin E, 25,000 IU; vitamin K3, $500.0 \mathrm{mg}$; vitamin B1, 5,000.0 mg; vitamin B2, 4,000.0 mg; vitamin B6; $10.0 \mathrm{mg}$; nicotinic acid, $15,000.0 \mathrm{mg}$; pantothenic acid, $10,000.0 \mathrm{mg}$; biotin, $150.0 \mathrm{mg}$; folic acid, $1,250.0 \mathrm{mg}$; vitamin C, $25,000.0 \mathrm{mg}$; cholin, 50,000.0 mg; inositol, 20,000.0 mg; iron 2,000.0 mg; copper, 3,500.0 mg; chelate copper, 1,500,0 mg; zinc, 10,500.0 mg; chelate zinc, $4,500.0 \mathrm{mg}$; manganese, 4,000.0 mg; selenium, $15.0 \mathrm{mg}$; chelate selenium, $15.0 \mathrm{mg}$; iodine, $150.0 \mathrm{mg}$; cobalt, $30.0 \mathrm{mg}$; chromium $80.0 \mathrm{mg}$; filler, $1,000.0 \mathrm{~g}$.

${ }^{8}$ Aquavi ${ }^{\circledR}$ Lys: $507 \mathrm{~g} \mathrm{~kg}^{-1}$ lysine (Evonik Nutrition \& Care GmbH, Hanau, Germany).

${ }^{9}$ Aquavi ${ }^{\circledR}$ Met-Met, DL-methionyl-DL-methionine, $950 \mathrm{~g} \mathrm{~kg}^{-1}$ methionine (Evonik Nutrition \& Care GmbH, Hanau, Germany).

${ }^{10}$ ThreAMINO $^{\circledR}, 985 \mathrm{~g} \mathrm{~kg}^{-1}$ threonine (Evonik Nutrition \& Care GmbH, Hanau, Germany).

${ }^{11}$ Sigma-Aldrich Co., $985 \mathrm{~g} \mathrm{~kg}^{-1}$ arginine (St. Louis, USA).

${ }^{12}$ Nutri-Bind Aqua Veg Dry, Nutri-Ad International NV (Dendermonde, Belgium).

${ }^{13}$ Cholesterol SF, $910 \mathrm{~g} \mathrm{~kg}^{-1}$ cholesterol (Dishman Netherlands B.V., Veenendaal, the Netherlands)

${ }^{14}$ Rovimix $^{\circledR}$ Stay $C^{\circledR} 35,350$ g kg-1 $^{-1}$ phosphorylated vitamin C (DSM Produtos Nutricionais Brasil Ltda., São Paulo, Brazil). 
made from polypropylene with $1.14 \mathrm{~m}$ of inner diameter on the bottom, $0.74 \mathrm{~m}$ height, and a bottom area of $1.02 \mathrm{~m}^{2}$. The system was exposed to weather conditions and a natural light cycle $(12 \mathrm{~h}$ light starting at $5.45 \mathrm{~h}$ ). In all tanks, continuous aeration was provided by an air diffusing system made with $0.5-\mathrm{m}$ aeration tubing (Aero-Tube ${ }^{\mathrm{TM}}$, Tekni-Plex Aeration, Austin, Texas, USA) rested near the bottom of each tank, but opposed to the feed delivery point. The system was supported by two 7.5-hp air blowers. All incoming water was filtered through a $240-\mathrm{kg}$ sand filter. Feed remains and shrimp feces acted as natural fertilizers, reducing water transparency progressively during shrimp culture. No additional fertilizers were needed. Water was exchanged at a rate of $100 \mathrm{~mL}$ second $^{-1}$ (14.4\% a day).

Tanks were provided with continuous aeration to reach near saturation of dissolved oxygen. Water temperature, salinity, and $\mathrm{pH}$ remained relatively stable during culture, at $27.4 \pm 0.56{ }^{\circ} \mathrm{C}(\mathrm{n}=4,452)$, $36 \pm 0.9 \mathrm{~g} \mathrm{~L}^{-1}(\mathrm{n}=4,452)$, and $8.23 \pm 0.19(\mathrm{n}=4,452)$, respectively. Total ammonia nitrogen (TAN),

Table 2 - Dry matter, crude protein, and amino acid composition of experimental diets

\begin{tabular}{|c|c|c|c|c|c|c|}
\hline \multirow{2}{*}{ Nutrient composition } & \multicolumn{5}{|c|}{ Diet composition ( $\mathrm{g} \mathrm{kg}^{-1}$ of the diet, dry matter basis) } & \multirow{2}{*}{ CV (\%) } \\
\hline & 4.6 & 5.6 & 6.9 & 7.9 & 9.2 & \\
\hline Dry matter & 902.3 & 907.9 & 908.1 & 911.2 & 912.5 & 0.43 \\
\hline Crude protein & 319.1 & 314.6 & 317.3 & 318.4 & 318.6 & 0.57 \\
\hline \multicolumn{7}{|c|}{ Essential amino acids (EAA) } \\
\hline Arginine & 20.6 & 20.4 & 20.5 & 20.9 & 20.5 & 0.80 \\
\hline Histidine & 6.7 & 6.6 & 6.7 & 6.7 & 6.6 & 0.58 \\
\hline Isoleucine & 12.4 & 12.1 & 12.1 & 12.3 & 12.0 & 1.32 \\
\hline Leucine & 21.3 & 21.0 & 21.1 & 21.2 & 20.8 & 0.87 \\
\hline Lysine & 19.3 & 19.4 & 19.7 & 20.0 & 20.1 & 1.94 \\
\hline Methionine & 4.6 & 5.6 & 6.9 & 7.9 & 9.2 & 26.54 \\
\hline Met+Cys ${ }^{1}$ & 8.9 & 10.0 & 11.2 & 12.1 & 13.5 & 15.99 \\
\hline Phenylalanine & 14.5 & 14.4 & 14.5 & 14.5 & 14.2 & 0.94 \\
\hline Threonine & 13.9 & 13.7 & 13.9 & 14.0 & 13.9 & 0.71 \\
\hline Tryptophan & 3.4 & 3.4 & 3.4 & 3.4 & 3.4 & 0.41 \\
\hline Valine & 13.3 & 13.1 & 13.0 & 13.1 & 13.0 & 0.98 \\
\hline Sum EAA & 139.9 & 139.7 & 142.9 & 146.0 & 147.3 & 2.60 \\
\hline \multicolumn{7}{|c|}{ Non-essential amino acids (NEAA) } \\
\hline Alanine & 12.2 & 12.0 & 12.1 & 12.1 & 11.9 & 0.79 \\
\hline Cystine & 4.3 & 4.4 & 4.3 & 4.2 & 4.3 & 1.40 \\
\hline Glycine & 13.7 & 13.6 & 13.6 & 13.7 & 13.4 & 0.85 \\
\hline Serine & 14.0 & 13.7 & 13.9 & 13.9 & 13.7 & 0.88 \\
\hline Proline & 19.8 & 19.6 & 19.6 & 19.7 & 19.2 & 1.22 \\
\hline Aspartate & 26.3 & 25.7 & 25.9 & 26.1 & 25.7 & 0.98 \\
\hline Glutamine & 61.7 & 61.1 & 61.0 & 61.5 & 60.4 & 0.77 \\
\hline Sum NEAA & 151.9 & 150.1 & 150.4 & 151.2 & 148.7 & 0.81 \\
\hline Total AA & 290.9 & 289.8 & 293.3 & 297.2 & 296.0 & 1.09 \\
\hline \multicolumn{7}{|l|}{ Free AA } \\
\hline Methionine & $<0.1$ & 0.2 & 0.3 & 0.4 & 0.3 & 20.56 \\
\hline Lysine & 5.6 & 5.8 & 6.1 & 6.1 & 6.5 & 6.36 \\
\hline Threonine & 3.6 & 3.8 & 3.9 & 3.9 & 4.1 & 4.28 \\
\hline Valine & $<0.2$ & $<0.2$ & $<0.2$ & $<0.2$ & $<0.2$ & - \\
\hline DL-Met-Met & $<0.1$ & 0.8 & 1.8 & 2.8 & 4.0 & 58.62 \\
\hline
\end{tabular}

${ }^{1}$ TSAA - total sulfur amino acids. 
nitrite $\left(\mathrm{NO}_{2}^{-}\right)$, and nitrate $\left(\mathrm{NO}_{3}^{-}\right)$remained below toxic values for penaeid shrimp, at $0.04 \pm 0.03 \mathrm{mg} \mathrm{L}^{-1}$ ( $\mathrm{n}=160), 0.30 \pm 0.29 \mathrm{mg} \mathrm{L}^{-1}(\mathrm{n}=160)$, and $1.25 \pm 0.69 \mathrm{mg} \mathrm{L}^{-1}(\mathrm{n}=160)$, respectively.

Shrimp were fed under two feed allowances, regular and in excess (Table 3). The excess feed allowance assumed a $30 \%$ increase in feed inputs relative to the regular allowance. The regular feed allowance was based on the maximum amount of feed (MM, g) that can be eaten daily by one individual of a specific body weight (BW), in accordance to the formula $\mathrm{MM}=0.0931 \mathrm{BW}^{0.6200}$ (Nunes and Parsons, 2000; Nunes et al., 2006; Façanha et al., 2018). Under the regular feeding regime, no feed restriction was imposed to meet desired FCR.

On the first 14 days of rearing, meals were adjusted on a daily basis following an estimated weight gain of $100 \mathrm{mg}$ day shrimp $^{-1}$ and a $0.5 \%$ weekly drop in shrimp survival across all diets. Biweekly (days 15, 30,45 , and 60 of rearing), rations were corrected by weighing individually five animals per tank. Until the following weight check, feed ration was adjusted according to calculated daily weight shrimp gains for each tank maintaining a $0.5 \%$ weekly drop in survival. Shrimp were fed daily at 7.00, 10.00, 13.00, and $16.00 \mathrm{~h}$, exclusively in feeding trays $(14.3 \times 3.5 \mathrm{~cm}$, diameter $\times$ height $)$, positioned at 1 unit per tank. Feeding trays were inspected daily to check for dead animals. In this case, dead shrimp was collected and subtracted from the initial stocked population. No dead animals were replaced throughout the culture period. All uneaten feed observed in feeding trays was collected, oven-dried, and weighted to calculate the apparent feed intake.

At harvest, all animals were counted and weighed individually to determine their final survival (\%), body weight (g), weekly growth (g), and gained yield $\left(\mathrm{g} \mathrm{m}^{-2}\right)$. Feed conversion ratio and apparent feed intake (AFI, $g$ of feed delivered divided by the number of stocked shrimp) were determined on a DM basis. Calculations followed equations presented by Nunes et al. (2019b).

The effect of dietary Met content, feed allowance, and their interaction on shrimp performance were analyzed through two-way ANOVA. The following mathematical model was adopted:

$$
Y i j=\mu+\tau i+\beta j+\gamma i j+\varepsilon i j
$$

in which $\mu$ is the overall mean response, $\tau i$ is the effect due to the $i$-th level of Met content $(4.6,5.6,6.9$, 7.9 , and $9.2 \mathrm{~g} \mathrm{~kg}^{-1}$ of the diet), $\beta \mathrm{j}$ is the effect due to the $j$-th level of feed allowance (regular and $30 \%$ in excess), and $\gamma$ ij is the effect due to any interaction between the $i$-th level of Met content and the $j$-th level of feed allowance. When significant differences were detected, they were compared two-by-two with Tukey's HSD test. The significant level of $5 \%$ was set in all statistical analyses. The statistical package SPSS 15.0 for Windows (SPSS Inc., Chicago, Illinois, USA) was used.

Table 3 - Feeding table used to adjust meals offered to L. vannamei juveniles reared under two feed allowances

\begin{tabular}{|c|c|c|c|c|}
\hline \multicolumn{2}{|c|}{ Shrimp body weight (g) } & \multicolumn{2}{|c|}{ Feeding rate ( $\%$ body weight) } & \multirow{2}{*}{$\begin{array}{l}\text { Shrimp weight gain } \\
\left(\mathrm{mg} \mathrm{day}^{-1}\right)\end{array}$} \\
\hline Initial & Final & Regular & Excess $^{1}$ & \\
\hline 1.50 & 3.00 & 7.06 & 9.17 & 100 \\
\hline 3.00 & 4.00 & 5.82 & 7.56 & 110 \\
\hline 4.00 & 5.00 & 5.27 & 6.86 & 120 \\
\hline 5.00 & 6.00 & 4.88 & 6.35 & 130 \\
\hline 6.00 & 7.00 & 4.58 & 5.95 & 140 \\
\hline 7.00 & 8.00 & 4.33 & 5.63 & 150 \\
\hline 8.00 & 9.00 & 4.13 & 5.37 & 160 \\
\hline 9.00 & 10.00 & 3.96 & 5.15 & 170 \\
\hline 10.00 & 11.00 & 3.81 & 4.96 & 180 \\
\hline 11.00 & 12.00 & 3.68 & 4.79 & 190 \\
\hline
\end{tabular}

${ }^{1} 30 \%$ increase in feed rations relative to a regular feed allowance. 


\section{Results}

A final shrimp survival of $86.5 \pm 3.6 \%$ (mean \pm standard error) was reached with no significant influence of feed allowance and dietary methionine (Met) content $(\mathrm{P}>0.05)$. However, feed inputs had a statistically significant effect on $\mathrm{AFI}$, weekly shrimp growth, final $\mathrm{BW}$, and gained yield $(\mathrm{P}<0.05$; Table 4$)$. Apparent feed intake was significantly higher when feed was delivered in excess compared with the regular feeding regime; it also increased progressively with higher levels of dietary Met, peaking at $6.9 \mathrm{~g} \mathrm{~kg}^{-1}$. Larger meals and a higher dietary Met had no significant impact on protein efficiency ratio (PER) and FCR, which reached a mean of $1.41 \pm 0.10$ and $2.75 \pm 0.16$, respectively.

There was a significant impact on shrimp growth as a result of feed allowance $(\mathrm{P}<0.05)$. As lower amounts of feed were delivered, shrimp grew slower, from an average of $0.78 \pm 0.04$ to $0.59 \pm 0.04 \mathrm{~g}$. As a result, there was an increment in gained yield by feeding shrimp $30 \%$ in excess $\left(789 \pm 42 \mathrm{~g} \mathrm{~m}^{-2}\right)$ compared with a regular feed allowance $\left(617 \pm 78 \mathrm{~g} \mathrm{~m}^{-2}\right)$.

A combined effect of feed allowance and dietary Met content was observed in shrimp final BW (Two-Way ANOVA; $\mathrm{P}<0.05$; Figure 1). Feeding shrimp with the lowest dietary Met content $\left(4.6 \mathrm{~g} \mathrm{~kg}^{-1}\right)$ in combination with regular feed inputs resulted in the lowest shrimp BW. By feeding animals $30 \%$ in excess, final BW was enhanced, and a peak was observed at $6.9 \mathrm{~g} \mathrm{~kg}^{-1}$ Met. Comparatively, a dietary Met content of $7.9 \mathrm{~g} \mathrm{~kg}^{-1}$ was required to achieve a maximum BW under a regular feed allowance. Thus, shrimp required less amounts of dietary Met to maximize BW when higher feed inputs were delivered. No further amounts of dietary Met were necessary to enhance BW under the conditions adopted.

Table 4 - Growth performance and feed utilization of the juvenile L. vannamei reared at $120 \mathrm{shrimp}^{-2}$ in an outdoor green-water system under a regular feed allowance and 30\% in excess

\begin{tabular}{|c|c|c|c|c|c|c|}
\hline \multirow{2}{*}{ Performance } & \multirow{2}{*}{$\begin{array}{c}\text { Feed } \\
\text { allowance }\end{array}$} & \multicolumn{5}{|c|}{ Dietary methionine (Met; $\mathrm{g} \mathrm{kg}^{-1}$ of the diet, DM) } \\
\hline & & 4.6 & 5.6 & 6.9 & 7.9 & 9.2 \\
\hline \multirow{2}{*}{ Final survival (\%) } & Regular & $87.7 \pm 2.7$ & $88.7 \pm 3.2$ & $86.9 \pm 1.5$ & $85.4 \pm 2.8$ & $89.3 \pm 3.0$ \\
\hline & Excess & $91.4 \pm 1.8$ & $84.2 \pm 3.5$ & $81.8 \pm 7.1$ & $84.4 \pm 5.7$ & $83.1 \pm 4.4$ \\
\hline \multirow{2}{*}{ Weekly growth (g) } & Regular & $0.54 \pm 0.03$ & $0.57 \pm 0.03$ & $0.60 \pm 0.02$ & $0.65 \pm 0.06$ & $0.58 \pm 0.04$ \\
\hline & Excess & $0.71 \pm 0.02$ & $0.77 \pm 0.03$ & $0.84 \pm 0.08$ & $0.76 \pm 0.04$ & $0.80 \pm 0.01$ \\
\hline \multirow{2}{*}{ Gained yield ${ }^{1}\left(\mathrm{~g} \mathrm{~m}^{-2}\right)$} & Regular & $566 \pm 56$ & $608 \pm 37$ & $628 \pm 35$ & $662 \pm 42$ & $619 \pm 20$ \\
\hline & Excess & $790 \pm 29$ & $782 \pm 60$ & $798 \pm 30$ & $769 \pm 40$ & $791 \pm 49$ \\
\hline \multirow{2}{*}{ AFI ( $g$ of feed shrimp ${ }^{-1}$ ) } & Regular & $12.4 \pm 0.2$ & $12.7 \pm 0.2$ & $13.0 \pm 0.2$ & $13.1 \pm 0.2$ & $12.8 \pm 0.2$ \\
\hline & Excess & $17.2 \pm 0.2$ & $17.5 \pm 0.3$ & $18.2 \pm 0.3$ & $18.0 \pm 0.2$ & $17.7 \pm 0.1$ \\
\hline \multirow{2}{*}{ PER } & Regular & $1.35 \pm 0.07$ & $1.42 \pm 0.07$ & $1.46 \pm 0.03$ & $1.56 \pm 0.12$ & $1.42 \pm 0.08$ \\
\hline & Excess & $1.29 \pm 0.03$ & $1.41 \pm 0.04$ & $1.45 \pm 0.11$ & $1.33 \pm 0.06$ & $1.41 \pm 0.02$ \\
\hline \multirow{2}{*}{ FCR } & Regular & $2.82 \pm 0.25$ & $2.66 \pm 0.17$ & $2.61 \pm 0.11$ & $2.51 \pm 0.14$ & $2.60 \pm 0.07$ \\
\hline & Excess & $2.74 \pm 0.09$ & $2.86 \pm 0.24$ & $2.88 \pm 0.16$ & $2.94 \pm 0.17$ & $2.84 \pm 0.17$ \\
\hline Two-way ANOVA & \multicolumn{2}{|c|}{$\mathrm{g} \mathrm{kg}^{-1}$ Methionine } & \multicolumn{2}{|c|}{ Feed allowance } & \multicolumn{2}{|c|}{$\mathrm{g} \mathrm{kg}^{-1}$ Met vs Feed allowance } \\
\hline Survival & \multicolumn{2}{|c|}{0.680} & \multicolumn{2}{|c|}{0.331} & \multicolumn{2}{|c|}{0.670} \\
\hline Growth & \multicolumn{2}{|c|}{0.217} & \multicolumn{2}{|c|}{$<0.0001$} & \multicolumn{2}{|c|}{0.862} \\
\hline Gained yield & \multicolumn{2}{|c|}{0.919} & \multicolumn{2}{|c|}{$<0.0001$} & \multicolumn{2}{|c|}{0.763} \\
\hline AFI & \multicolumn{2}{|c|}{0.004} & \multicolumn{2}{|c|}{$<0.0001$} & \multicolumn{2}{|c|}{0.756} \\
\hline PER & \multicolumn{2}{|c|}{0.203} & \multicolumn{2}{|c|}{0.570} & \multicolumn{2}{|c|}{0.390} \\
\hline FCR & \multicolumn{2}{|c|}{0.995} & \multicolumn{2}{|c|}{0.060} & \multicolumn{2}{|c|}{0.650} \\
\hline
\end{tabular}

AFI - apparent feed intake; FCR - feed conversion ratio; PER - protein efficiency ratio.

${ }^{1}$ Final shrimp biomass(g) subtracted from initial shrimp biomass (g), divided by tank area $\left(\mathrm{m}^{-2}\right)$.

Two-way analysis of variance indicated statistical differences between dietary Met levels and feed allowance and their interaction $(\mathrm{P}<0.05)$ Initial shrimp body weight $=1.83 \pm 0.14 \mathrm{~g}(\mathrm{n}=5,376)$. Data represent the means \pm standard error of means. 


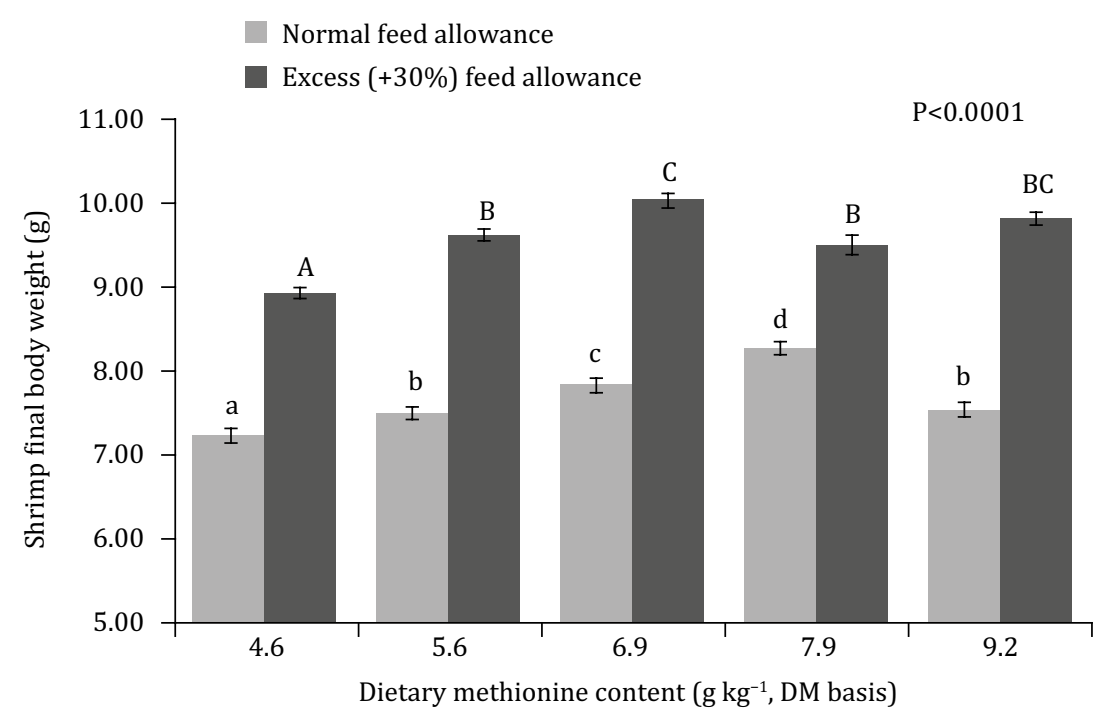

Different lowercase and uppercase letters indicate statistical differences between dietary methionine levels within normal and excess feed allowance, respectively, at the $\alpha=0.05$ level according to Tukey's HSD.

Figure 1 - Mean ( \pm standard error) body weight ( $\mathrm{g}$ ) of L. vannamei after 70 days of rearing in green-water tanks of $1 \mathrm{~m}^{3}$.

\section{Discussion}

Feed allowance and dietary amino acid content are important factors that influence nutrient intake and retention (Cho and Bureau, 2001) and, consequently, shrimp growth (Venero et al., 2007; Richard et al., 2011). In our study, there was a significant interaction of feed inputs and dietary Met content with the growth performance of L. vannamei. In a green-water rearing system, an increase in feed inputs allowed for a reduction in total Met content in a low-fish meal diet without compromising the growth of juvenile shrimp. Final body weight of L. vannamei was maximized with $6.9 \mathrm{~g} \mathrm{~kg}^{-1} \mathrm{Met}_{\text {when shrimp }}$ was fed $30 \%$ in excess or with $7.9 \mathrm{~g} \mathrm{~kg}^{-1}$ under a regular feeding regime. Thus, up to a certain level, as more feed was delivered and consumed, the less dietary Met was required to achieve higher BW and vice-versa. This implies shrimp is dependent on a minimum daily intake of dietary Met to maximize its growth performance.

Some investigations have shown that feed allowance can be reduced while increasing the nutrient density without affecting animal growth or yield (Cho et al., 2001; Venero et al., 2007). Most of the published studies on the reduction of feed allowance has adopted the dietary protein and energy ratio approach (Cho et al., 2001; Cho and Lovell, 2002; Kureshy and Davis, 2002). In the present study, the nutrient density varied only in regards to the dietary Met content. According to Kureshy and Davis (2002), L. vannamei fed different dietary protein levels present variations in their optimal performance for protein. For juvenile and subadult shrimp, maximum growth of L. vannamei fed a $320 \mathrm{~g} \mathrm{~kg}^{-1} \mathrm{protein}$ diet was 7 and 15\% higher than when fed a $480 \mathrm{~g} \mathrm{~kg}^{-1}$ protein diet, respectively. Venero et al. (2007) also observed that higher protein-density diets allowed for a reduction of feed allowance without affecting the growth performance of L. vannamei. Our findings corroborate these studies. A lower feed allowance can be compensated by a higher dietary Met concentration and vice-versa. Therefore, the dietary nutrient density and feed allowance must be adjusted proportionally.

Our data indicate that a $30 \%$ increase in feed inputs with $6.9 \mathrm{~g} \mathrm{~kg}^{-1}$ Met was more advantageous for the growth performance of L. vannamei than a reduction in feed allowance with higher levels of Met. Raising feed inputs resulted in a significantly higher yield without deteriorating shrimp survival and feed efficiency. An economic analysis would be required to define which approach is more cost-competitive. Previous studies on L. vannamei could not establish a relation between dietary amino acid levels, final survival, and feed efficiency (Millamena et al., 1996; Xie et al., 2012; Zhou et al., 2012; Liu et al., 2014; Façanha et al., 2016). As expected for a green-water system, additional sources of natural food in the 
rearing environment can further improve feed efficiency (Venero et al., 2007) and shrimp survival, while reducing the amount of dietary Met needed to achieve maximum growth performance (Façanha et al. 2016). Nunes et al. (2006) observed that shrimp survival is not affected by different feed allowances even under experimental conditions different from the present study, including rearing water (clear), feeding rates (in excess and 25,50 , and $75 \%$ rate-restricted), stocking density ( 36 shrimp $\mathrm{m}^{-2}$ ), water exchange rate (129.6\% per day), and dietary nutrient composition.

Other work showed that increasing feed allowance does not enhance shrimp performance. In a recent study, Ullman et al. (2019) found that increasing feed rations by $15 \%$ when feeding twice a day results in no advantage to L. vannamei performance. Shrimp were fed a $375 \mathrm{~g} \mathrm{~kg}^{-1} \mathrm{CP}$ feed, but the amino acid composition was not reported. Although feed input was variable, CP content was constant throughout their study. Our findings do not imply that a higher feed allowance is required to enhance shrimp growth performance. Nevertheless, they indicate that feed allowance and dietary Met (Met+Cys) composition interact and should be considered when determining daily meals.

Commercial operations adjust feed inputs based on weekly shrimp weight gains and estimates of shrimp survival, feed intake, and FCR. Farmers often use the CP content as an indicator of feed performance and amino acid (AA) composition. As dietary CP may derive from protein sources deficient or (and) with poor bioavailability of essential AA, including Met (Nunes et al., 2014), CP alone may not provide the accuracy required to assess feed quality. Instead, choosing feeds based on the composition of essential AA could assist in determining feed performance and if higher or lower doses of feed inputs are required to maximize shrimp growth and yield. Therefore, shrimp farmers should adjust feed inputs based on feed nutrient quality and density rather than growth performance indicators alone.

In our study, increasing the amount of feed delivered led to a higher AFI. These results are similar to those obtained for Penaeus monodon by Allan et al. (1995). The authors reported that AFI was significantly higher when a high feeding rate was adopted. Since shrimp have small stomachs, feed intake in penaeid shrimp can occur while an earlier meal is still being digested. Nunes and Parsons (2000) reported that in juvenile Farfantepenaeus subtilis, food load occurred progressively as more feed was given and evacuated from stomach, while feeding continued at reduced levels. Satiation is suggested to be controlled by the loading capacity of their digestive gland, where final digestion and absorption of nutrients take place. Our experimental diets likely contained similar levels of digestible energy, so AFI was limited by energy satiation.

In the present study, effects of dietary Met and feed allowance levels on proximate composition of whole shrimp body and muscle after the feeding trial were not analyzed. Under similar rearing conditions, Façanha et al. (2016) was not able to detect any significant differences in the content of CP, Met, total sulfur amino acids, and sum of essential amino acids in the muscle, hepatopancreas, and natural food of L. vannamei fed graded levels of dietary Met during 10 weeks of rearing.

The different shrimp performance outcomes in our study indicate that exogenous variables (e.g., natural food availability, stocking density), other than the animal alone, have an impact on the level of dietary Met that should be targeted for maximum growth. It was also possible to determine that shrimp yield and growth can be affected by both feed allowance and dietary Met content. Shrimp grow slower, and lower yields are achieved when feed and Met allowance are restricted. This will occur regardless of the dietary Met content.

\section{Conclusions}

There is a sparing effect of dietary methionine for L. vannamei when feed inputs are increased. Thus, whiteleg shrimp appear to have an absolute daily requirement for dietary methionine based on ration size. Higher feed allowance compensates for lower levels of dietary methionine, allowing shrimp to reach larger body weights without any significant cost to feed efficiency. This is explained by the fact that higher amounts of feed translate into a higher allowance of dietary Met (methionine + cysteine) and other nutrients. 


\section{Conflict of Interest}

The authors declare no conflict of interest.

\section{Author Contributions}

Conceptualization: C. Figueiredo-Silva and A.J.P. Nunes. Data curation: A.J.P. Nunes. Formal analysis: F.N. Façanha and A.J.P. Nunes. Funding acquisition: A.R. Oliveira-Neto, C. Figueiredo-Silva and A.J.P. Nunes. Investigation: F.N. Façanha, H. Sabry-Neto, A.R. Oliveira-Neto, C. Figueiredo-Silva and A.J.P. Nunes. Methodology: F.N. Façanha, A.R. Oliveira-Neto, C. Figueiredo-Silva and A.J.P. Nunes. Project administration: F.N. Façanha, H. Sabry-Neto, A.R. Oliveira-Neto and A.J.P. Nunes. Resources: A.J.P. Nunes. Software: A.J.P. Nunes. Supervision: H. Sabry-Neto, A.R. Oliveira-Neto, C. Figueiredo-Silva and A.J.P. Nunes. Validation: A.R. Oliveira-Neto. Writing-original draft: F.N. Façanha and A.J.P. Nunes. Writingreview \& editing: A.R. Oliveira-Neto, C. Figueiredo-Silva and A.J.P. Nunes.

\section{Acknowledgments}

The first author received a scholarship from Fundação Cearense de Apoio ao Desenvolvimento Científico e Tecnológico (FUNCAP, Edital 03/2014). The last author acknowledges the support from a research productivity fellowship (CNPq/MCTIC, PQ\# 303678/2017-8).

\section{References}

Allan, G. L.; Moriarty, D. J. W. and Maguire, G. B. 1995. Effects of pond preparation and feeding rate on production of Penaeus monodon Fabricius, water quality, bacteria and benthos in model farming ponds. Aquaculture 130:329-349. https://doi.org/10.1016/0044-8486(94)00316-G

AOAC - Association of Official Analytical Chemists. 2002. Official methods of analysis of the Association of Official Analytical Chemists International. 16th ed. AOAC, Arlington, VA, USA.

Cho, S. H.; Jo, J. Y. and Kim, D. S. 2001. Effects of variable feed allowance with constant energy and ratio of energy to protein in a diet for constant protein input on the growth of common carp (Cyprinus carpio L.). Aquaculture Research 32:349-356. https://doi.org/10.1046/j.1365-2109.2001.00564.x

Cho, C. Y. and Bureau, D. P. 2001. A review of diet formulation strategies and feeding systems to reduce excretory and feed wastes in aquaculture. Aquaculture Research 32:349-360. https://doi.org/10.1046/j.1355-557x.2001.00027.x

Cho, S. H. and Lovell, R. T. 2002. Variable feed allowance with constant protein input for channel catfish (Ictalurus punctatus) cultured in ponds. Aquaculture 204:101-112. https://doi.org/10.1016/S0044-8486(01)00645-7

Duy, H. N.; Coman, G. J.; Wille, M.; Wouters, R.; Quoc, H. N.; Vu, T.; Kim, D. T.; Van, H. N. and Sorgeloos, P. 2012. Effect of water exchange, salinity regime, stocking density and diets on growth and survival of domesticated black tiger shrimp Penaeus monodon (Fabricius, 1798) reared in sand-based recirculating systems. Aquaculture 338-341:253-259. https://doi.org/10.1016/j.aquaculture.2012.01.021

Façanha, F. N.; Oliveira-Neto, A. R.; Figueiredo-Silva, C. and Nunes, A. J. P. 2016. Effect of shrimp stocking density and graded levels of dietary methionine over the growth performance of Litopenaeus vannamei reared in a green-water system. Aquaculture 463:16-21. https://doi.org/10.1016/j.aquaculture.2016.05.024

Façanha, F. N.; Sabry-Neto, H.; Figueiredo-Silva, C.; Oliveira-Neto, A. R. and Nunes, A. J. P. 2018. Minimum water exchange spares the requirement for dietary methionine for juvenile Litopenaeus vannamei reared under intensive outdoor conditions. Aquaculture Research 49:1682-1689. https://doi.org/10.1111/are.13624

Figueiredo-Silva, C.; Lemme, A.; Sangsue, D. and Kiriratnikom, S. 2015. Effect of DL-methionine supplementation on the success of almost total replacement of fish meal with soybean meal in diets for hybrid tilapia (Oreochromis niloticus $\times$ Oreochromis mossambicus). Aquaculture Nutrition 21:234-241.

Kureshy, N. and Davis, D. A. 2002. Protein requirement for maintenance and maximum weight gain for the Pacific white shrimp, Litopenaeus vannamei. Aquaculture 204:125-143. https://doi.org/10.1016/S0044-8486(01)00649-4

Liu, F. J.; Liu, Y. J.; Tian, L. X.; Li, X. F.; Zhang, Z. H.; Yang, H. J. and Du, Z. Y. 2014. Quantitative dietary isoleucine requirement of juvenile Pacific white shrimp, Litopenaeus vannamei (Boone) reared in low-salinity water. Aquaculture International 22:1481-1497. https://doi.org/10.1007/s10499-014-9761-y 
Millamena, O. M.; Bautista-Teruel, M. N. and Kanazawa, A. 1996. Methionine requirement of juvenile tiger shrimp Penaeus monodon Fabricius. Aquaculture 143:403-410. https://doi.org/10.1016/0044-8486(96)01270-7

NRC - National Research Council. 2011. Nutrient requirements of fish and shrimp. NRC, Washington, DC, USA.

Nunes, A. J. P.; Gesteira, T. C. V. and Goddard, S. 1997. Food ingestion and assimilation by the Southern brown shrimp Penaeus subtilis under semi-intensive culture in NE Brazil. Aquaculture 149:121-136. https://doi.org/10.1016/S00448486(96)01433-0

Nunes, A. J. P. and Parsons, G. J. 2000. Size-related feeding and gastric evacuation measurements for the Southern brown shrimp Penaeus subtilis. Aquaculture 187:133-151. https://doi.org/10.1016/S0044-8486(99)00386-5

Nunes A. J. P.; Sá, M. V. C.; Carvalho, E. A. and Sabry-Neto, H. 2006. Growth performance of the white shrimp Litopenaeus vannamei reared under time-and rate-restriction feeding regimes in a controlled culture system. Aquaculture 253:646-652. https://doi.org/10.1016/j.aquaculture.2005.09.023

Nunes, A. J. P.; Sá, M. V. C. and Sabry-Neto, H. 2011. Growth performance of the white shrimp, Litopenaeus vannamei, fed on practical diets with increasing levels of the Antarctic krill meal, Euphausia superba, reared in clear-versus green-water culture tanks. Aquaculture Nutrition 17:e511-e520. https://doi.org/10.1111/j.1365-2095.2010.00791.x

Nunes, A. J. P.; Sá, M. V. C.; Browdy, C. L. and Vázquez-Añón, M. 2014. Practical supplementation of shrimp and fish feeds with crystalline amino acids. Aquaculture 431:20-27. https://doi.org/10.1016/j.aquaculture.2014.04.003

Nunes, A. J. P.; Sabry-Neto, H. and Masagounder, K. 2019a. Crude protein in low-fish meal diets for juvenile Litopenaeus vannamei can be reduced through a well-balanced supplementation of essential amino acids. Journal of the World Aquaculture Society 1-15. https://doi.org/10.1111/jwas.12605

Nunes, A. J. P.; Sabry-Neto, H.; Silva, F. H. P.; Oliveira-Neto, A. R. and Masagounder, K. 2019b. Multiple feedings enhance the growth performance and feed efficiency of juvenile Litopenaeus vannamei when fed a low-fish meal amino acidsupplemented diet. Aquaculture International 27:337-347. https://doi.org/10.1007/s10499-018-0330-7

Richard, L.; Vachot, C.; Surget, A.; Rigolet, V.; Kaushik, S. J. and Geurden, I. 2011. The effect of choline and cystine on the utilisation of methionine for protein accretion, remethylation and trans-sulfuration in juvenile shrimp Penaeus monodon. British Journal of Nutrition 106:825-835. https://doi.org/10.1017/S0007114511001115

Venero, J. A.; Davis, D. A. and Rouse, D. B. 2007. Variable feed allowance with constant protein input for the pacific white shrimp Litopenaeus vannamei reared under semi-intensive conditions in tanks and ponds. Aquaculture 269:490-503. https://doi.org/10.1016/j.aquaculture.2007.02.055

Ullman, C.; Rhodes, M.; Hanson, T.; Cline, D. and Davis, D. A. 2019. Effects of four different feeding techniques on the pond culture of Pacific white shrimp, Litopenaeus vannamei. Journal of the World Aquaculture Society 50:54-64. https://doi. org/10.1111/jwas.12531

Xie, F. J.; Zeng, W. P.; Zhou, Q. C.; Wang, H. L.; Wang, T.; Zheng, C. Q. and Wang, Y. L. 2012. Dietary lysine requirement of juvenile Pacific white shrimp, Litopenaeus vannamei. Aquaculture 358-359:116-121. https://doi.org/10.1016/j. aquaculture.2012.06.027

Zhou, Q. C.; Zeng, W. P.; Wang, H. L.; Wang, T.; Wang, Y. L. and Xie, F. J. 2012. Dietary arginine requirement of juvenile Pacific white shrimp, Litopenaeus vannamei. Aquaculture 364-365:252-258. https://doi.org/10.1016/j.aquaculture.2012.08.020 